\title{
ETHNOMEDICINAL USES OF PLANTS AMONG THE NEWAR COMMUNITY OF PHARPING VILLAGE OF KATHMANDU DISTRICT, NEPAL
}

\author{
N.P. Balami
}

\begin{abstract}
The present paper highlights 119 species of plants used as medicine by the Newar community of Pharping village of Kathmandu district. All reported medicinal plants were used for 35 types of diseases like Diabetes, Epilepsy, Fever, Jaundice, Rheumatism and other condition such as incense, spice and flavourant etc.
\end{abstract}

district.

Key words: Ethnomedicine, Newar, Pharping village, Kathmandu

\section{INTRODUCTION}

Nepal occupies one third of Himalayas lying at $80^{\circ} 04^{\prime}$ to $88^{\circ} 12^{\prime} \mathrm{E}$ and $26^{\circ} 22^{\prime}$ to $30^{\circ} 27^{\prime} \mathrm{N}$ in meeting point of Central Himalayas and Eastern Himalayas .Nepal has rich floral diversity due to high altitudinal, topographic, climatic and edaphic variations, so that various types of forest are found. The different ethnic groups are traditionally linked to resources available in the forest Ethnobotany refers to the study of the interaction between people and plants (Martin, 1995).There is inseparable interrelationship between the ethnic groups and plants. However due to changing perception of the local people, commercialization and socio-economic transformation of all over the world, it has been observed that the indigenous knowledge on resource use has been degraded (Silori \& Rana, 2000).

In Nepal, the concept of ethnomedicine has been developed since the late $19^{\text {th }}$ century (1885-1901 A.D). The first book "Chandra-Nighantu regarding medical plants was published by the Royal Nepal Academy in 1969 (2025 B.S.). Later, a number of ethnobotanical studies on different ethnic groups of Nepal have been carried out by different workers (Pandey, 1964; Malla \& Shakya, 1968; Adhikari \& Shakya, 1977; Sacherer, 1979; Malla \& Shakya, 1984-1985; Manandhar, 1985, 1990b, 1994-1995; Shrestha \& Pradhan, 1986-1993; Joshi et al. 1998; Shrestha et al., 1998; Chhetri, 1999; Singh, 1999; Shakya et al., 1999; Ghimire et al., 2000; IUCN, 2000; Joshi \& Joshi, 2001; Rajbhandari, 2001and Balami, 2003).

The study area is situated in Kathmandu district $\left(27^{0} 34^{\prime}\right.$ to $27^{0} 38^{\prime} \mathrm{N}$ latitude and $85^{\circ} 12^{\prime}$ to $85^{\circ} 16^{\prime} \mathrm{E}$ longitude) of Bagmati zone in the mid hills of central Nepal. It has sub-tropical climate. Schima-Castanopsis, Pinus roxburghii and Alnus nepalensis are important forest types at the lower belt and broad leaved 
oak forest (Quercus glauca, Q. lanata, Q. semecarpifolia etc) are observed at upper belt. The annual temperature $24^{0} \mathrm{C}$ and receives $1161 \mathrm{~mm}$ rainfall annually

Among different community, Newar is one of the major community in Pharping Village. During the time of Malla, this village was called "Shikharapur Nagari". It was a South door of Kathmandu valley for entrance.

\section{MATERIALS AND METHODS}

Herbarium specimens were critically examined and identified with help of the relevant literatures (HMG/N, 1969; Hooker, 1872-1897; Hara et al., 1978; Hara \& Williams, 1979; Hara et al., 1982; Stainton \& Polunin, 1984; Stainton, 1988 and Press et al., 2000). Ethno medicinal information was gathered from experienced persons, local faith healers and traditional medicine practitioners. Voucher specimens have been deposited in Tribhuvan University Central Herbarium (TUCH) for preservation. Although 119 species of plants used as medicine by Newar of the Pharping village of the Kathmandu district have been arranged in alphabetical order with botanical name in italics. Name of the family and local names have been given in parenthesis, followed by parts used and ethnomedicinal uses of plants as gathered from local people during the field visits.

\section{ENUMERATION OF SPECIES}

\section{HeRBS}

\begin{tabular}{|c|l|l|}
\hline 1 & $\begin{array}{l}\text { Achyranthes aspera L. (Amaranthaceae, } \\
\text { Dattiwan) }\end{array}$ & Lf juice for stomach ache. \\
\hline 2 & $\begin{array}{l}\text { Aconitum ferox Wall. ex Ser. } \\
\text { (Ranunculaceae, Bikhma) }\end{array}$ & Rh powder for insecticide. \\
\hline 3 & $\begin{array}{l}\text { Allium wallichi Kunth ( } \\
\text { Amaryllidaceae,Ban laba) }\end{array}$ & $\begin{array}{l}\text { Wp raw for spice and } \\
\text { flavourant and appetizer. }\end{array}$ \\
\hline 4 & $\begin{array}{l}\text { Amaranthus spinosus } \\
\text { L.(Amaranthaceae) }\end{array}$ & Rt liquid for urinary trouble. \\
\hline 5 & $\begin{array}{l}\text { Anaphalis contorta } \text { (D. Don) Hook. f. } \\
\text { (Compositae, Bho swan) }\end{array}$ & Rt liquid for diarrhoea. \\
\hline 6 & $\begin{array}{l}\text { Anaphalis margaritacea } \text { (L.) Benth. } \\
\text { (Compositae, Bho swan) }\end{array}$ & Rt liquid for diarrhoea. \\
\hline 7 & $\begin{array}{l}\text { Anaphalis triplinervis (Sims) C.B. } \\
\text { Clarke (Compositae, Bho swan) }\end{array}$ & Rt liquid for diarrhoea. \\
\hline 8 & $\begin{array}{l}\text { Anemone vitifolia } \text { Buch.-Ham. ex DC. } \\
\text { (Ranunculaceae) }\end{array}$ & Wp juice for antileech. \\
\hline 9 & $\begin{array}{l}\text { Anemone rivularis Buch.-Ham. ex } \\
\text { DC.(Ranunculaceae) }\end{array}$ & Wp juice for antileech. \\
\hline 10 & $\begin{array}{l}\text { Arisaema erubescens (Wall.) Schott } \\
\text { (Araceae, Taha ya kani) }\end{array}$ & Rh raw/liquid for anthelmintic. \\
\hline 11 & $\begin{array}{l}\text { Arisaema nepenthoides (Wall.) Mart. ex } \\
\text { Schott (Araceae, Taha ya kani) }\end{array}$ & Rh raw/liquid for anthelmintic. \\
\hline
\end{tabular}


TRIBHUVAN UNIVERSITY JOURNAL, VOL. , NO.

\begin{tabular}{|c|c|c|}
\hline 12 & $\begin{array}{l}\text { Arisaema tortuosum (Wall.) Schott. } \\
\text { (Araceae, Taha ya kani) }\end{array}$ & Rh raw/liquid for anthelmintic. \\
\hline 13 & $\begin{array}{l}\text { Asclepias curassavica } \\
\text { L.(Asclepiadaceae) }\end{array}$ & Wp liquid for antiseptic. \\
\hline 14 & $\begin{array}{l}\text { Asparagus racemosus var. subacerosus } \\
\text { Baker (Liliaceae, Kurilo) }\end{array}$ & $\begin{array}{l}\text { Rh raw/powder for aphrodisiac, } \\
\text { diabetes and tonic }\end{array}$ \\
\hline 15 & $\begin{array}{l}\text { Asparagus racemosus Willd. var. } \\
\text { recemosus (Liliaceae, Kurilo) }\end{array}$ & $\mathrm{Rh}$ raw/powder for diabetes. \\
\hline 16 & $\begin{array}{l}\text { Astilbe rivularis Buch.-Ham. ex D. Don } \\
\text { (Saxifragaceae, Facu waasha) }\end{array}$ & $\begin{array}{l}\text { Rh raw/powder for aphrodisiac } \\
\text { and meternity problems. }\end{array}$ \\
\hline 17 & $\begin{array}{l}\text { Begonia nepalensis (A. DC.) Warb. } \\
\text { (Begoniaceae) }\end{array}$ & Rt liquid for anthelmintic. \\
\hline 18 & $\begin{array}{l}\text { Bergenia ciliata forma ligulata Yeo } \\
\text { (Saxifragaceae, Pakhaanbed) }\end{array}$ & $\begin{array}{l}\text { Rh raw/powder/liquid for } \\
\text { aphrodisiac, Fever, Meternity } \\
\text { problem and post pregnancy. }\end{array}$ \\
\hline 19 & $\begin{array}{l}\text { Brachycorythis obcordata (Lindl.) } \\
\text { Summerh. (Orchidaceae, Gamdol) }\end{array}$ & $\begin{array}{l}\text { Rh raw/powder for aphrodisiac } \\
\text { and tonic. }\end{array}$ \\
\hline 20 & Caltha palustris L. (Ranunculaceae) & Lf juice for fever. \\
\hline 21 & $\begin{array}{l}\text { Centella asiatica }(\mathrm{L} .) \text { Urb. } \\
\text { (Umbelliferae, Kholcha ghain) }\end{array}$ & $\begin{array}{l}\text { Wp juice for antidote to poison, } \\
\text { cut and wounds and urinary } \\
\text { trouble. }\end{array}$ \\
\hline 22 & $\begin{array}{l}\text { Cirsium verutum (D. Don) Spreng. } \\
\text { (Compositae, Chwackan) }\end{array}$ & Rt liquid for urinary trouble. \\
\hline 23 & $\begin{array}{l}\text { Cissampelos pareira L. } \\
\text { (Menispermaceae, Batulo pate) }\end{array}$ & Wp juice for haemorrhage. \\
\hline 24 & $\begin{array}{l}\text { Clematis buchananiana DC. } \\
\text { (Ranunculaceae, Gwya swan) }\end{array}$ & Lf liquid for cough and cold. \\
\hline 25 & $\begin{array}{l}\text { Cuscuta europaea var. indica Engelm. } \\
\text { (Convolvulaceae, Mhasu lahara) }\end{array}$ & Wp liquid for jaundice. \\
\hline 26 & $\begin{array}{l}\text { Cuscuta reflexa Roxb. } \\
\text { (Convolvulaceae, Mhasu lahara) }\end{array}$ & Wp liquid for jaundice. \\
\hline 27 & $\begin{array}{l}\text { Cyanoglossum zeylanicum (Vahl ex } \\
\text { Hornem.) Thunb. ex Lehm. } \\
\text { (Boraginaceae, Khokan ghain) }\end{array}$ & Wp juice for cut and wounds. \\
\hline 28 & $\begin{array}{l}\text { Cyathula tomentosa (Roth) Moq. } \\
\text { (Amaranthaceae, Khokan ma) }\end{array}$ & Rt decoction for fever. \\
\hline 29 & $\begin{array}{l}\text { Cynodon dactylon (L.) Pers. } \\
\text { (Gramineae, Guntun ma) }\end{array}$ & Wp liquid for haemorrhage. \\
\hline 30 & $\begin{array}{l}\text { Cyperus rotundus L. (Cyperaceae, Kusa } \\
\text { ghain) }\end{array}$ & Rt liquid for fever. \\
\hline 31 & $\begin{array}{l}\text { Delphinium cooperi Munz } \\
\text { (Ranunculaceae) }\end{array}$ & $\mathrm{Rh}$ raw/liquid for fever. \\
\hline 32 & $\begin{array}{l}\text { Didymocarpus leucocalyx C.B. Clarke } \\
\text { (Gesneriaceae, Kum kum) }\end{array}$ & Wp powder for incense. \\
\hline 33 & Dioscorea bulbifera L. (Dioscoreaceae, & Rh powder/liquid for throat \\
\hline
\end{tabular}


ETHNOMEDICINAL USES OF PLANTS AMONG THE NEWAR

\begin{tabular}{|c|c|c|}
\hline & Phahi) & sore. \\
\hline 34 & $\begin{array}{l}\text { Dioscorea deltoidea Wall. ex Griseb. } \\
\text { (Dioscoreaceae, Vyakur) }\end{array}$ & $\begin{array}{l}\text { Rh powder/liquid for } \\
\text { anthelmintic }\end{array}$ \\
\hline 35 & $\begin{array}{l}\text { Dipsacus inermis Wall. Var. inermis } \\
\text { (Dipsacaceae, Ban lain) }\end{array}$ & Rt powder for insectcide. \\
\hline 36 & $\begin{array}{l}\text { Drymaria cordata (L.) Willd. ex Roem. } \\
\& \text { Schult. (Caryophyllaceae, Kaacha } \\
\text { ghain) }\end{array}$ & $\begin{array}{l}\text { Wp juice for cut, wounds and } \\
\text { eye ailment. }\end{array}$ \\
\hline 37 & $\begin{array}{l}\text { Eclipta prostata (L.) L. (Compositae, } \\
\text { Antalcha) }\end{array}$ & Wp raw for anthelmintic. \\
\hline 38 & $\begin{array}{l}\text { Euphorbia heterophylla L. } \\
\text { (Euphorbiaceae, Duruo ghain) }\end{array}$ & St liquid for cut and wounds \\
\hline 39 & $\begin{array}{l}\text { Euphorbia hirta L. (Euphorbiaceae, } \\
\text { Duruo ghain) }\end{array}$ & Wp liquid for antiseptic. \\
\hline 40 & $\begin{array}{l}\text { Fagopyrum debotrys (D. Don) H. Hara } \\
\text { (Polygonaceae, Faaku waasha ma) }\end{array}$ & $\begin{array}{l}\text { Rh powder/liquid for stomach } \\
\text { ache }\end{array}$ \\
\hline 41 & $\begin{array}{l}\text { Galium elegans Wall. ex Roxb. } \\
\text { (Rubiaceae) }\end{array}$ & Wp liquid for antiseptic. \\
\hline 42 & $\begin{array}{l}\text { Gerbera maxima (D. Don) Beauverd } \\
\text { (Compositae, Jhule) }\end{array}$ & Rt powder/liquid for antiseptic. \\
\hline 43 & $\begin{array}{l}\text { Girardinia diversifolia (Link) Friis } \\
\text { (Urticaceae, Dya nhakan) }\end{array}$ & Bd raw for diabetes. \\
\hline 44 & $\begin{array}{l}\text { Hedychium spicatum Sm. } \\
\text { (Zingiberaceae, Pani sarro) }\end{array}$ & $\begin{array}{l}\text { Rh decoction/liquid for cough } \\
\text { and cold. }\end{array}$ \\
\hline 45 & $\begin{array}{l}\text { Heracleum nepalense D. Don } \\
\text { (Umbelliferae, Ban jii) }\end{array}$ & $\begin{array}{l}\text { Fr raw/powder for spice and } \\
\text { flavourant. }\end{array}$ \\
\hline 46 & $\begin{array}{l}\text { Hydrocotyl javanica Thunb. } \\
\text { (Umbelliferae) }\end{array}$ & Lf juice for ear pain. \\
\hline 47 & $\begin{array}{l}\text { Imperata cylindrica (L.) P. Beauv. } \\
\text { (Gramineae, Kasi ghain) }\end{array}$ & $\begin{array}{l}\text { Rt powder/liquid for } \\
\text { anthelmintic. }\end{array}$ \\
\hline 48 & $\begin{array}{l}\text { Iris decora Wall. (Iridaceae, Tarwal } \\
\text { swan) }\end{array}$ & Rt. Liquid for constipation. \\
\hline 49 & $\begin{array}{l}\text { Lobelia pyramidalis Wall. } \\
\text { (Cmpanulaceae, Akalbir) }\end{array}$ & Wp powder for insecticide. \\
\hline 50 & $\begin{array}{l}\text { Oxalis corniculata L. (Oxalidaceae, } \\
\text { Paauncha ghain) }\end{array}$ & Wp raw for appetizer. \\
\hline 51 & $\begin{array}{l}\text { Pedicularis bifida (Buck.-Ham. ex D. } \\
\text { Don) }\end{array}$ & $\begin{array}{l}\text { Pennell (Scrophulariaceae) } \\
\text { Rt liquid for stomach ache }\end{array}$ \\
\hline 52 & $\begin{array}{l}\text { Pedicularis gracilis Wall. ex Benth. } \\
\text { (Scrophulariaceae) }\end{array}$ & Rt liquid for stomach ache \\
\hline 53 & $\begin{array}{l}\text { Pimpinella diversifolia } \mathrm{DC} . \\
\text { (Umbelliferae) }\end{array}$ & $\begin{array}{l}\text { Fr decoction for cough and } \\
\text { cold. }\end{array}$ \\
\hline 54 & Plantago erosa Wall. (Plantaginaceae) & Sd raw for constipation. \\
\hline
\end{tabular}


TRIBHUVAN UNIVERSITY JOURNAL, VOL. , NO.

\begin{tabular}{|c|c|c|}
\hline 55 & $\begin{array}{l}\text { Polygonatum verticilatum (L.) All. } \\
\text { (Liliaceae,) }\end{array}$ & Ys raw for tonic. \\
\hline 56 & $\begin{array}{l}\text { Potentilla fulgens var. intermedia Hook. } \\
\text { f. (Rosaceae,Bajradanti) }\end{array}$ & Rt powder/raw for toothache. \\
\hline 57 & $\begin{array}{l}\text { Roscoea purpurea } \mathrm{Sm} . \\
\text { (Zingiberaceae,Local panch aunle) }\end{array}$ & $\begin{array}{l}\text { Rh raw /powder for } \\
\text { aphrodisiac. }\end{array}$ \\
\hline 58 & $\begin{array}{l}\text { Rubia manjith Roxb. ex Fleming } \\
\text { (Rubiaceae,Majitho) }\end{array}$ & $\begin{array}{l}\text { Rt liquid/paste for antiseptic } \\
\text { and rheumatism. }\end{array}$ \\
\hline 59 & $\begin{array}{l}\text { Satyrium nepalense D. Don } \\
\text { (Orchidaceae) }\end{array}$ & Rt juice for fever. \\
\hline 60 & Scutellaria discolor Colebr. (Labiatae) & Rt raw/powder for fever. \\
\hline 61 & $\begin{array}{l}\text { Selinum tenuifolium Wall. ex C.B. } \\
\text { Clarke (Umbelliferae) }\end{array}$ & Wp liquid for cough and cold. \\
\hline 62 & Solanum nigrum L. (Solanaceae) & Fr raw for diabetes. \\
\hline 63 & Solidago virga-aurea L. (Compositae) & Wp liquid for diarrhoea. \\
\hline 64 & $\begin{array}{l}\text { Sonchus arvensis L. (Compositae, } \\
\text { Khaiken ma) }\end{array}$ & Wp raw for diabetes. \\
\hline 65 & $\begin{array}{l}\text { Spilanthes calva DC. (Compositae, Lata } \\
\text { ghain) }\end{array}$ & Wp liquid for toothache. \\
\hline 66 & $\begin{array}{l}\text { Spiranthes sinensis var. amoena (M. } \\
\text { Bieb.) H. Hara (Orchidaceae) }\end{array}$ & Rh raw/powder for aphrodisiac. \\
\hline 67 & $\begin{array}{l}\text { Swertia angustifolia Buch.-Ham. ex D. } \\
\text { Don (Gentianaceae, Khalu ma) }\end{array}$ & Wp liquid for fever. \\
\hline 68 & $\begin{array}{l}\text { Swertia chirayita (Roxb. ex Fleming) } \\
\text { H. Karst. (Gentianaceae, Khalu ma) }\end{array}$ & $\begin{array}{l}\text { Wp raw/liquid for } \\
\text { anthelmintic, diarrhoea, fever, } \\
\text { headache and stomach ache. }\end{array}$ \\
\hline 69 & $\begin{array}{l}\text { Swertia nervosa (G. Don) C.B. Clarke } \\
\text { (Gentianaceae, Khalu ma) }\end{array}$ & Wp liquid for fever. \\
\hline 70 & $\begin{array}{l}\text { Thalictrum virgatum Hook. f. \& } \\
\text { Thomson (Ranunculaceae) }\end{array}$ & Rt liquid for toothache. \\
\hline 71 & $\begin{array}{l}\text { Tinospora sinensis (Lour.) Merr. } \\
\text { (Menispermaceae, Gurjo gana) }\end{array}$ & $\begin{array}{l}\text { Rh juice/powder/liquid for } \\
\text { diabetes and gastritis. }\end{array}$ \\
\hline 72 & $\begin{array}{l}\text { Trichosanthes lepiniana }=T \text {. } \\
\text { tricuspidata Lour. (Cucurbitaceae, } \\
\text { Indrayani) }\end{array}$ & Sd raw for throat sore. \\
\hline 73 & Urtica dioica L. (Urticaceae, Nhakan) & $\mathrm{Bd} / \mathrm{Ys}$ raw for diabetes. \\
\hline 74 & $\begin{array}{l}\text { Valeriana hardwickii Wall. } \\
\text { (Valerianaceae, Chwamaycha) }\end{array}$ & Wp powder for incense. \\
\hline 75 & $\begin{array}{l}\text { Valeriana wallichi }=V . \text { jatamansi Jones } \\
\text { (Valerianaceae, Chwamaycha) }\end{array}$ & Wp powder for incense. \\
\hline 76 & Viola serpens Wall. (Violaceae) & Wp liquid for cough \& cold. \\
\hline
\end{tabular}

SHRUB 
ETHNOMEDICINAL USES OF PLANTS AMONG THE NEWAR

\begin{tabular}{|c|c|c|}
\hline 1 & $\begin{array}{l}\text { Artemisia dubia Wall. ex Besser } \\
\text { (Compositae, Dhuswan) }\end{array}$ & $\begin{array}{l}\text { Wp/Lf juice for antileech, cut } \\
\& \text { wound. }\end{array}$ \\
\hline 2 & $\begin{array}{l}\text { Artemisia indica Willd. (Compositae, } \\
\text { Dhuswan) }\end{array}$ & $\begin{array}{l}\mathrm{Wp} / \mathrm{Lf} \text { juice /liquid for } \\
\text { antileech and indigestion. }\end{array}$ \\
\hline 3 & $\begin{array}{l}\text { Berberis aristata DC. (Berberidaceae, } \\
\text { Ban marpasi) }\end{array}$ & Rt juice for stomach ache. \\
\hline 4 & $\begin{array}{l}\text { Berberis asiatica Roxb. ex DC. } \\
\text { (Berberidaceae, Marpasi) }\end{array}$ & Rt juice for stomach ache. \\
\hline 5 & $\begin{array}{l}\text { Cannabis sativa L. (Cannabaceae, } \\
\text { Gagima) }\end{array}$ & $\mathrm{Sd}$ raw/paste for diarrhoea. \\
\hline 6 & $\begin{array}{l}\text { Cinnamomum tamala (Buch.-Ham.) } \\
\text { Nees \& Eberm. (Lauraceae, Tej pat) }\end{array}$ & $\begin{array}{l}\text { Br/Lf raw/powder for spice } \\
\text { and flavourant. }\end{array}$ \\
\hline 7 & $\begin{array}{l}\text { Coriaria nepalensis Wall. } \\
\text { (Coriariaceae, Hakupasima) }\end{array}$ & Lf liquid for antiseptic. \\
\hline 8 & $\begin{array}{l}\text { Daphne papyracea Wall. ex steud. } \\
\text { (Thymelaeaceae, Lokta) }\end{array}$ & $\begin{array}{l}\text { Rt liquid for antidote to poison } \\
\text { and fever. }\end{array}$ \\
\hline 9 & $\begin{array}{l}\text { Dichroa febrifuga Lour. } \\
\text { (Hydrangeaceae) }\end{array}$ & $\begin{array}{l}\text { Rt liquid for antidote to poison } \\
\text { and fever. }\end{array}$ \\
\hline 10 & $\begin{array}{l}\text { Elsholtzia flava (Benth.) Benth. } \\
\text { (Labiatae, Ban tumo) }\end{array}$ & Lf juice paste for insect bite. \\
\hline 11 & $\begin{array}{l}\text { Eupatorium chinense L. (Compositae, } \\
\text { Ban mara) }\end{array}$ & Lf juice for cut and wound. \\
\hline 12 & $\begin{array}{l}\text { Eupatorium adenophorum Spreng. } \\
\text { (Compositae, Ban mara) }\end{array}$ & $\begin{array}{l}\mathrm{Wp} / \mathrm{Lf} \text { liquid/juice for } \\
\text { antiseptic, cut and wound. }\end{array}$ \\
\hline 13 & $\begin{array}{l}\text { Gaultheria fragrantissima Wall. } \\
\text { (Ericaceae, Dhaisikacha) }\end{array}$ & Wp liquid for rheumatism. \\
\hline 14 & $\begin{array}{l}\text { Inula cappa (Buch.-Ham. ex D. Don) } \\
\text { DC. (Compositae, Kan pate) }\end{array}$ & $\begin{array}{l}\text { Rt liquid/dicoction for } \\
\text { epilepsy and rheumatism. }\end{array}$ \\
\hline 15 & $\begin{array}{l}\text { Justicia adhatoda L. } \\
\text { (Acanthaceae,Aaleha) }\end{array}$ & Inf/Lf liquid for fever. \\
\hline 16 & $\begin{array}{l}\text { Lindera pulcherrima (Nees) Benth. ex } \\
\text { Hook. f. (Lauraceae, Siltimur) }\end{array}$ & $\begin{array}{l}\mathrm{Br} / \mathrm{Lf} \text { raw/powder for spice } \\
\text { and flavourant. }\end{array}$ \\
\hline 17 & $\begin{array}{l}\text { Maesa chisia Buch.-Ham. ex D. Don } \\
\text { (Myrsinaceae, Bilauni) }\end{array}$ & Br powder for insecticide. \\
\hline 18 & $\begin{array}{l}\text { Mahonia nepaulensis DC. } \\
\text { (Berberidaceae, Jamane Mandro) }\end{array}$ & $\begin{array}{l}\mathrm{Fr} / \mathrm{Br} \text { juice for diarrhoea and } \\
\text { dysentery. }\end{array}$ \\
\hline 19 & $\begin{array}{l}\text { Osbeckia nepalensis Hook. } \\
\text { (Melastomataceae) }\end{array}$ & $\begin{array}{l}\mathrm{Fl} / \mathrm{Lf} \text { paste for cut and } \\
\text { wounds. }\end{array}$ \\
\hline 20 & $\begin{array}{l}\text { Osbeckia stellata Buch.-Ham. ex D. } \\
\text { Don.(Melastomataceae) }\end{array}$ & $\begin{array}{l}\mathrm{Fl} / \mathrm{Lf} \text { paste for cut and } \\
\text { wounds. }\end{array}$ \\
\hline 21 & $\begin{array}{l}\text { Phyllanthus emblica L. } \\
\text { (Euphorbiaceae, Amo/Dyapasi) }\end{array}$ & $\mathrm{Fr} / \mathrm{Sd}$ raw for cough and cold. \\
\hline 22 & $\begin{array}{l}\text { Prinsepia utilis Royle. (Rosaceae, } \\
\text { Dhatelo) }\end{array}$ & $\begin{array}{l}\text { Fr oil for body pain, joint pain } \\
\text { and rheumatism. }\end{array}$ \\
\hline 23 & Ricinus communis L. (Euphorbiaceae, & Lf/Fr dicoction/liquid for \\
\hline
\end{tabular}


TRIBHUVAN UNIVERSITY JOURNAL, VOL. , NO.

\begin{tabular}{|c|c|c|}
\hline & Ala ma) & $\begin{array}{l}\text { body pain and antidote to } \\
\text { snake bite. }\end{array}$ \\
\hline 24 & $\begin{array}{l}\text { Rubus ellipticus Sm. (Rosaceae, } \\
\text { Yelsima) }\end{array}$ & Rt liqud for anthelmintic. \\
\hline 25 & $\begin{array}{l}\text { Sarcococca coriacea (Hook.) Sweet } \\
\text { (Buxaceae) }\end{array}$ & $\begin{array}{l}\text { Lf liquid for scabies and skin } \\
\text { diseases. }\end{array}$ \\
\hline 26 & $\begin{array}{l}\text { Smilax aspera L. (Liliaceae, } \\
\text { Kukurdyno) }\end{array}$ & $\begin{array}{l}\text { St/Lf liquid for meternity } \\
\text { problems/post pregnancy \& } \\
\text { scabies and skin diseases. }\end{array}$ \\
\hline 27 & $\begin{array}{l}\text { Smilax lanceifolia Roxb. (Liliaceae, } \\
\text { Kukurdyno) }\end{array}$ & Rt powder for insecticide. \\
\hline 28 & $\begin{array}{l}\text { Solanum aculeatissimum Jacq. } \\
\text { (Solanaceae, Kantakari) }\end{array}$ & Fr/Rt liquid for jaundice. \\
\hline 29 & $\begin{array}{l}\text { Solanum pseudo-capsicum L. } \\
\text { (Solanaceae) }\end{array}$ & Fr raw for diabetes. \\
\hline 30 & $\begin{array}{l}\text { Thysanolaena maxima (Roxb.) Kuntze } \\
\text { (Gramineae, Tuphima) }\end{array}$ & Rt liquid for anthelmintic. \\
\hline 31 & $\begin{array}{l}\text { Vitex negundo L. (Verbenaceae, } \\
\text { Bosingali) }\end{array}$ & Lf powder for cough and cold. \\
\hline 32 & $\begin{array}{l}\text { Wordfordia fructicosa (L.) Kurz } \\
\text { (Lythraceae, Dhainyaro) }\end{array}$ & Fl juice for dysentery. \\
\hline \multirow[t]{2}{*}{33} & $\begin{array}{l}\text { Zanthoxylum armatum DC., (Rutaceae, } \\
\text { Timuma) }\end{array}$ & Fr juice/raw/powder for \\
\hline & $\begin{array}{l}\text { antileech,appetizer,indigestion, spice \& } \\
\text { flavourant. }\end{array}$ & \\
\hline 34 & $\begin{array}{l}\text { Zanthoxylum oxyphyllum Edgew. } \\
\text { (Rutaceae, Ban timuma) }\end{array}$ & $\mathrm{Lf} / \mathrm{Fr}$ juice for antileech. \\
\hline
\end{tabular}

\section{Tree}

\begin{tabular}{|c|l|l|}
\hline 1 & $\begin{array}{l}\text { Bauhinia purpurea } \text { L. (Leguminosae, } \\
\text { Ban kunopma) }\end{array}$ & $\begin{array}{l}\text { Fl raw for diarrhoea \& } \\
\text { dysentery. }\end{array}$ \\
\hline 2 & $\begin{array}{l}\text { Bauhinia variegata } \text { L. (Leguminosae, } \\
\text { Kunopma) }\end{array}$ & $\begin{array}{l}\text { Fl raw for diarrhoea \& } \\
\text { dysentery. }\end{array}$ \\
\hline 3 & $\begin{array}{l}\text { Lyonia ovalifolia } \text { (Wall.) Drude } \\
\text { (Ericaceae, Degdhyasima) Br paste for } \\
\text { scabies \& skin diseases. }\end{array}$ & $\begin{array}{l}\text { Melia azedarach L. (Meliaceae, } \\
\text { Khayubakasima) }\end{array}$ \\
\hline 5 & $\begin{array}{l}\text { Myrica esculanta } \text { Buch.-Ham. ex D. } \\
\text { Don (Myricaceae, Kapasima) Br } \\
\text { liquid for fever. }\end{array}$ & $\begin{array}{l}\text { Br/Sd juice /raw for } \\
\text { anthelmintic. }\end{array}$ \\
\hline 6 & $\begin{array}{l}\text { Nyctanthes arbor-tristis L. (Oleaceae, } \\
\text { Parijat) }\end{array}$ & Lf liquid for fever. \\
\hline 7 & $\begin{array}{l}\text { Rhus javanica } \text { L. (Anacardiaceae, } \\
\text { Bhamo chhasima) }\end{array}$ & Fr liquid for diarrhoea. \\
\hline
\end{tabular}


ETHNOMEDICINAL USES OF PLANTS AMONG THE NEWAR

\begin{tabular}{|c|l|l|}
\hline 8 & $\begin{array}{l}\text { Schima wallichi (DC.) Korth. } \\
\text { (Theaceae, Gwe chhasima) }\end{array}$ & Br powder for antiseptic. \\
\hline 9 & $\begin{array}{l}\text { Syzygium cumini (L.) Skeels } \\
\text { (Myrtaceae, Gulumusya ma) }\end{array}$ & Br juice for dysentery. \\
\hline
\end{tabular}

$(\mathrm{Br}=$ Bark, $\quad \mathrm{Bd}=\mathrm{Bud}, \quad \mathrm{Fl}=$ Flower, $\quad \mathrm{Fr}=$ Fruit, $\quad \mathrm{Inf}=$ Inflorescence, Lf=Leaf, $\mathrm{Rh}=$ Rhizome, $\mathrm{Rt}=$ Root, $\mathrm{Sd}=$ Seed, $\mathrm{St}=$ Stem, $\mathrm{Wp}=$ Whole plant, $\mathrm{Ys}=$ Young shoot)

\section{DISCUSSION}

Most of the plant species had multiple uses which were used in the treatment of 35 types of diseases and other conditions.

At the time of the ethnobotanical information of medicinal plant in study area, it was noted that a single disease, for example, Rheumatism was treated by number of different plant species such as Gaultheria fragrantissima, Inula cappa, Prinsepia utilis, Rubia manjith etc. Similarly a single plant like Swertia chirayita ,Bergenia ciliata, Asparagus racemosus or Astilbe rivularis were used to cure a number of diseases. For example, Swertia chirayita was used for treating headache, fever, anthelmintic and sometimes also used for diarrhoea. Among all the above mentioned 119 medicinal plant species, Asparagus racemosus, Astilbe rivularis, Bergenia ciliata forma ligulata and Swertia chirayita were most widely used. Similarly, popularly used other plant species were Eupatorium adenophorum, Centella asiatica, Drymeria cordata and Zanthoxylum armatum.

\section{WORKS CITED}

Adhikari, P.M. \& T.P. Shakya (1977). "Pharmacological Screening of Some Medicinal Plants of Nepal." J. Nep. Pharma, Assoc. 5 (1): 41-50.

Balami, N.P. (2003). Ethno-ecology of medicinal and aromatic plants of Kharpa Community Forest. M.Sc. Thesis, submitted to CDB, T.U., Kirtipur.

Chhetri, D.B. (1999). Diversity of Medicinal and Aromatic Plants in Manang, Central Nepal with Emphasis on Ecology and Essential Oil Variation of Jatamansi (Nardostachys grandiflora DC.). M.Sc. Thesis, submitted to CDB, T.U., Kirtipur, Nepal. Gurung, V.L. 1979. Medicinal Ferns of Nepal. J. Nep. Pharma Assoc. A Special Issue: 49-59.

Ghimire, S.K., Shrestha, A.K. Shrestha, K.K. \& P.K. Jha (2000). Plant resources use and human impact around Royal Bardia National Park, Nepal. J. Nat. His. Mus., 19: 3-26.

Hara, H. \& L.H.J. Williams. (1979). An Enumeration of the Flowering Plants of Nepal. Vol. II, London: Trustees of British Museum (Natural History).

Hara, H., Charter A.O. \& L.H.J. Williams (1982). An Enumeration of the Flowering Plants of Nepal. Vol. III, London: Trustees of British Museum (Natural History). 
TRIBHUVAN UNIVERSITY JOURNAL, VOL. , NO.

Hara, H., Stearn, W.T. \& L.H.J. Williams (1978). An Enumeration of Flowering Plants of Nepal. Vol. I, London: Trustees of British Museum (Natural History).

HMG/N (1969). Flora of Phulchoki and Godawari. Bulletin of the Department of Medicinal Plant No. 2. Kathmandu: Ministry of Forest, Department of Plant Resources, His Majesty's Government of Nepal.

Hooker, J.D. (1872-1897). The Flora of British India Vol. I-VII. London: L. Reeve and Co.

IUCN (2000). National Register of Medicinal Plants. The World Conservation Union (IUCN).

Joshi, K.K. \& S.D. Joshi (2001). Genetic Heritage of Medicinal and Aromatic Plants of Nepal Himalayas. Kathmandu: Buddha Academic Publishers and Distributors Pvt. Ltd.

Joshi, S., Vaidya, S. \& I. Shrestha (1998). Contribution to the Medicinal Flora of Pashupati Area of Kathmandu Valley. Research paper, submitted to Research Division, T U., Kirtipur.

Malla, S.B. \& P.R. Shakya (1984-85). Medicinal Plants of Nepal, In Nepal Nature's Paradise (T.C. Majupuria). Thailand. White Lotus Company, Bangkok.

Malla, S.B. \& P.R. Shakya (1968). Vegetation and Medicinal Plants of Nepal. Nepal National Commission for UNESCO. Regional Seminar on the Ecology of Tropical Highlands Organized by HMG and UNESCO, 8-16 April.

Manandhar, N.P. (1985). Ethnobotanical Notes on Certain Medicinal Plants used by Tharus of Dang-Deukhuri District, Nepal Int. J. Crude Drug Res., 23 (4): 153-259.

Manandhar, N.P. (1990b). Traditional Phytotherapy of Danuwar Tribes of Kamalakhonj in Sindhuli District, Nepal. Fitoterapia 61 (4): 325-331.

Manandhar, N.P. (1994). The ethnobotanical survey of herbal drugs of Kaski district, Nepal. Fitoterapia, 65 (1): 1-13.

Manandhar, N.P. (1995). Ethnobotanical notes on unexploited wild food plants of Nepal. Ethnobotany, 7 (1-2): 95-101.

Martin, G.J. (1995). Ethnobotany. A 'People and Plants' Conservation Manual. Chapman and Hall, London-Weinheim, New York, Tokyo, Melbourne and Madras.

Pandey, P.R. (1964). Distribution of Medicinal Plants in Nepal. Symposium on Medicinal Plants (Ceylon). 15-18 December.

Press, J.R, Shrestha, K.K. \& D.A. Sutton. 2000. Annotated Checklist of the Flowering Plants of Nepal. London: The Natural History Museum.

Rajbhandari, K.R. (2001). Ethnobotany of Nepal. Kathmandu: Ethnobotanical Society of Nepal (ESON). p. 189.

Sacherer, J. (1979). "The high altitude ethnobotany of the Rolwaling Sherpas." Contribution to Nepalese Studies, 4(2): 45-64. 
Shakya, M.R., Bajracharya, D.M., Joshi, G.P. \& J. Shakya (1999). Ethnobotany and plant diversity of Royal Shukla Phanta Wildlife Reserve, Nepal. In Proc. III Conf. Sci. \& Tech., Kathmandu: RONAST, pp. 288-295.

Shrestha, I. \& N. Pradhan (1986). Medicinal Plants of Chobhar Village of Kathmandu, Nepal. J. Nat. Hist. Mus. 10 (1-4): 65-72.

Shrestha, I. \& N. Pradhan (1993). Medicinal Plants of the Lele Village of Lalitpur, Nepal, Int. J. Pharmacog. 31 (2): 130-134.

Shrestha, K.K., Ghimire, S.K., Gurung, T.N., Lama, Y.C. \& Y. Aumeeruddy (1998). Conservation of Plant Resources, Community Development and Training in Applied Ethnobotany at Shey-Phoksundo National Park and its Bufferzone, Dolpa. WWF/Nepal Program Report Series No. 33.

Silori, C.S. \& A.R. Rana (2000). Indigenous knowledge on Medicinal Plants and Their Use in Narayan Sarovar Sanctuary, Kachchh, Ethnobotany, 12:1-7.

Singh, L.M. (1999). Medicinal plants of Nepal. An overview, In: Shrestha, R. and B. Shrestha (1999) (eds.) wild relatives of cultivated plants in Nepal. Proceedings of National Conference on Wild relatives of cultivated plants in Nepal, Kathmandu: The Green Energy Mission /Nepal.

Stainton, A. \& O. Polunin (1984). Flowers of Himalaya. New Delhi: Oxford University Press.

Stainton, A. (1988). Flower of the Himalaya a Supplement. New Delhi: Oxford University Press. 